\title{
Lung segmentation in CT for thoracic PET-CT registration through visual study
}

\begin{abstract}
Hybrid Positron Emission Tomography (PET)/Computer Tomography (CT) is a combined device that merges the anatomical and functional information of a patient used most commonly in cancer diagnosis and staging. This device is said to improve co-registration of both the anatomical and functional information. However, in real clinical practices, voluntary and involuntary patient motions are inevitable during scanning resulting in registration errors. In our study, feature based registration algorithm is to be used to solve such problem focusing on the thoracic region. Thus, segmentation method is chosen as a preliminary step to segment significant anatomical regions such as the lungs. Lungs segmentation in CT image is implemented based on optimal thresholding, region growing, connected component labeling as well as morphological operations. Satisfactory lung boundary segmentation results are obtained through visual inspection for our current dataset; that are deemed agreeable to devise a solution for PET-CT thoracic misregistration.
\end{abstract}

Keyword: Multi-modality PET-CT registration; CT lung segmentation; Threshold; Morphology; Region growing 